\title{
Intact cerebrovascular autoregulation in patients with refractory status epilepticus due to sufficient anesthetic treatment on a neurointensive care unit: a prospective cohort study
}

\author{
Marco Meyer ${ }^{1,2}$, Martin Juenemann ${ }^{2}$, Tobias Braun², Ingo Schirotzek ${ }^{2}$ and Patrick Schramm ${ }^{3,4^{*}}$ (D)
}

Status epilepticus (SE) is a serious emergency requiring immediate therapy to prevent severe seizure-related brain damage and secondary complications. Cerebrovascular autoregulation (CA) is a key component of cerebral hemostasis and is interictally compromised in epilepsy patients [1]. An impairment of CA may cause further neuronal damage due to fluctuations in cerebral perfusion pressure with consecutive cerebral edema or ischemia. We recently explored the time course of CA in patients with refractory SE and the need for deep analgo-sedation after failure of basic treatment with benzodiazepines and high-dose levetiracetam. All patients received analgo-sedation using propofol or midazolam and sufentanil with the aim of a burst-suppression pattern in electroencephalography. Mechanical ventilation was adapted to normocapnia and blood pressure to normal values. CA was calculated once daily for the first 4 days after the onset of SE by correlation of cerebral blood flow velocities (CBFVs) in both middle cerebral arteries measured with transcranial Doppler ultrasound and invasively measured arterial blood pressure. CA was expressed as the mean velocity index $(\mathrm{Mx})$ as previously described [2]. $\mathrm{Mx}$ is a variable with no defined cut-off, but $\mathrm{Mx}>0.3$ was associated with poor clinical outcomes in traumatic brain injury patients [3]. Ten adults (six male, four female) with refractory SE and a mean age of $52 \pm 16$ years were included (Table 1). Initially, Mx was $0.30 \pm 0.21$ and did not significantly change during the measurement period (Fig. 1 Table 2). Four patients were extubated and transferred to the regular ward after less than four measurements. The collected data indicated that CA in patients with refractory SE after induction of analgo-sedation was intact and did not change in a relevant matter during the observational time. Moreover, Mx levels $>0.3$ were not associated with poor clinical outcomes in the investigated cohort. Studies focusing on CA or CBFV in epilepsy patients are rare. In contrast to the presented data, a compromised CA was described in epilepsy patients in interictal states [1]. Furthermore, CBFV was increased during tonic-clonic seizures but was not observable during SE and nonconvulsive SE in comatose patients $[4,5]$. Neither an increased CBFV nor alterations of CA were found; this may be attributable to sufficient seizure treatment due to analgo-sedation. In conclusion, cerebral hemostasis seems to be preserved in sufficiently treated patients with refractory SE.

\footnotetext{
* Correspondence: schrammp@uni-mainz.de

${ }^{3}$ Department of Neurology, University Hospital Giessen and Marburg

Location Marburg, Baldingerstrasse, 35033 Marburg, Germany

${ }^{4}$ Department of Anesthesiology, Johannes Gutenberg-University, University

Medical Hospital Mainz, Langenbeckstr. 1, 55131 Mainz, Germany

Full list of author information is available at the end of the article
} 
Table 1 Physiological data

\begin{tabular}{ll}
\hline Age (years) & $52 \pm 16$ \\
Sex & 6 male, 4 female \\
BMI $\left(\mathrm{kg} / \mathrm{m}^{2}\right)$ & $27 \pm 6$ \\
$\mathrm{APACHE} \|$ & $22.8 \pm 3$ \\
$\mathrm{GOS} 4+5(n)$ & 6 \\
GOS $2+3(n)$ & 3 \\
GOS 1 ( $n)$ & 1 \\
ICU stay (days) & $11(9-18)$ \\
\hline
\end{tabular}

Parameters are expressed as mean \pm standard deviation or median (minimum-maximum)

APACHE II Acute Physiology and Chronic Health Evaluation II, BMI body mass index, GOS Glasgow Outcome Scale, ICU intensive care unit

Table 2 Mean velocity index and clinical data

\begin{tabular}{|c|c|c|c|c|c|c|c|c|}
\hline Day & $n$ & $M x$ & SOFA & MAP $(\mathrm{mmHg})$ & HR (beats/min) & $\mathrm{CBFV}_{\text {mean }}(\mathrm{cm} / \mathrm{s})$ & $\mathrm{PaO}_{2}(\mathrm{kPa})$ & $\mathrm{PaCO}_{2}(\mathrm{kPa})$ \\
\hline 1 & 10 & $0.30 \pm 0.21$ & $10.5 \pm 2.4$ & $89 \pm 14$ & $65 \pm 14$ & $42 \pm 15$ & $20.5 \pm 7.2$ & $4.9 \pm 0.7$ \\
\hline 2 & 9 & $0.34 \pm 0.35$ & $10.4 \pm 1.6$ & $84 \pm 16$ & $67 \pm 23$ & $55 \pm 22$ & $15.6 \pm 2.4$ & $5.1 \pm 1.1$ \\
\hline 3 & 8 & $0.28 \pm 0.34$ & $10.0 \pm 2.4$ & $90 \pm 11$ & $74 \pm 25$ & $53 \pm 23$ & $14.4 \pm 3.2$ & $5.3 \pm 0.9$ \\
\hline 4 & 6 & $0.32 \pm 0.45$ & $8.6 \pm 4.6$ & $97 \pm 16$ & $83 \pm 21$ & $56 \pm 23$ & $14.9 \pm 2,8$ & $6.9 \pm 1.0$ \\
\hline
\end{tabular}

The number of patients with refractory status epilepticus is presented as count $(n)$

$C B V F_{\text {mean }}$ mean cerebral blood flow velocity, $H R$ heart rate, $M A P$ mean arterial blood pressure, $M x$ mean velocity index, $\mathrm{PaCO}_{2}$ partial pressure of carbon dioxide, $\mathrm{PaO}_{2}$ partial pressure of oxygen, SOFA sequential organ failure assessment score

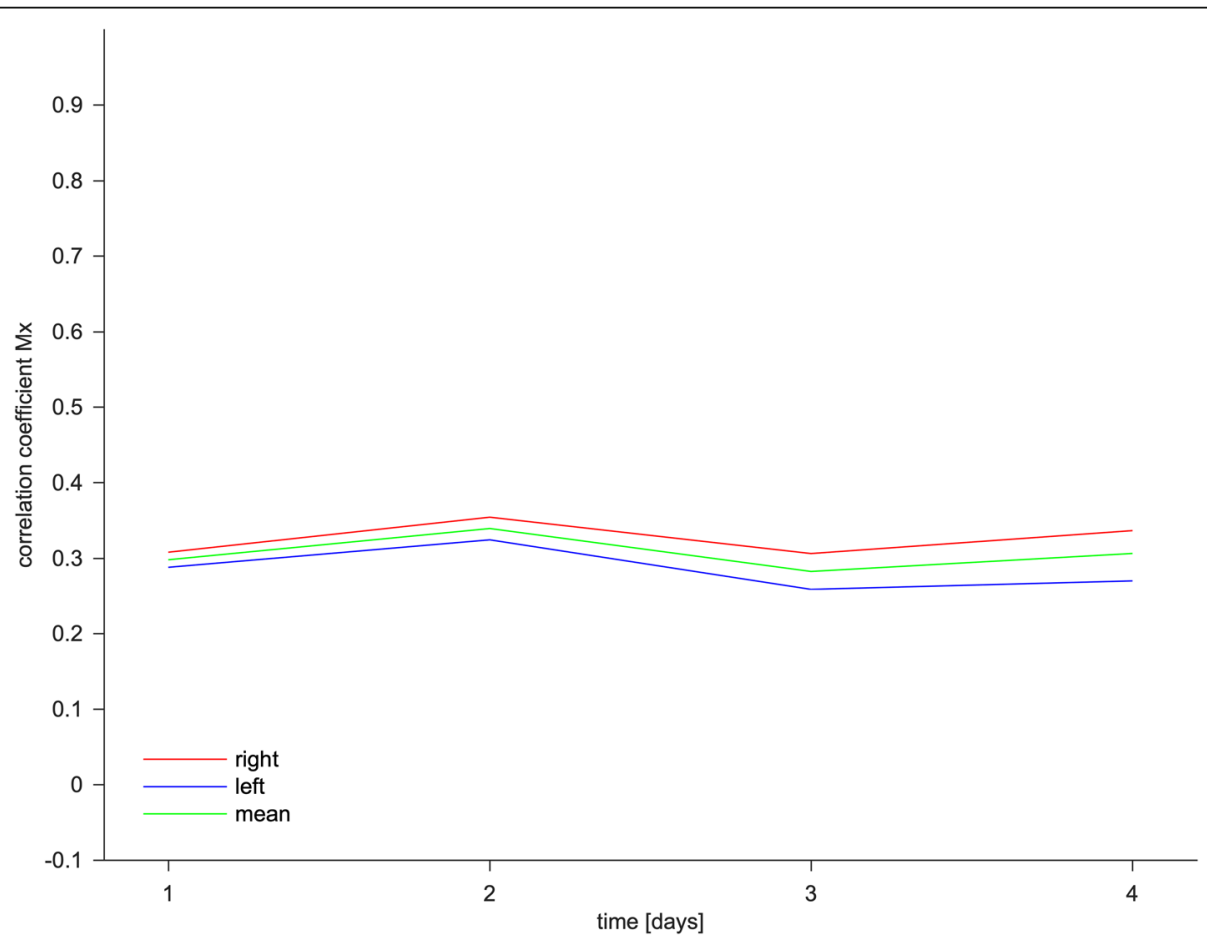

Fig. 1 Time course of cerebrovascular autoregulation index. The figure presents the index of the cerebrovascular autoregulation (mean velocity index; Mx) during the first 4 days after onset of status epilepticus. The red line represents $M x$ of the right hemisphere, the blue line represents the left hemisphere, and the yellow line represents mean $\mathrm{Mx}$ 


\section{Abbreviations}

CA: Cerebrovascular autoregulation; CBFV: Cerebral blood flow velocity; Mx: Mean velocity index; SE: Status epilepticus

\section{Acknowledgments}

None.

\section{Funding}

None.

\section{Availability of data and materials}

The datasets generated and/or analyzed during the current study are available from the corresponding author on reasonable request.

\section{Authors' contributions}

MM, IS, and PS: conceptualization. MM and PS: data collection. MM, MJ, TB, and PS: analysis of the data and statistics. MM and PS: preparation of original draft. All authors: review and editing. All authors read and approved the final manuscript.

\section{Ethics approval and consent to participate}

The present study was approved by the ethics committee of the Justus Liebig University, Giessen, Germany (No. 185/16). The informed consent of the patient or the legal representative was obtained before individual data were entered into the analysis.

\section{Consent for publication}

Not applicable.

\section{Competing interests}

The authors declare that they have no competing interests.

\section{Publisher's Note}

Springer Nature remains neutral with regard to jurisdictional claims in published maps and institutional affiliations.

\section{Author details}

${ }^{1}$ Department of Geriatric, Jung-Stilling Hospital Siegen, Wichernstrasse 40, 57074 Siegen, Germany. ${ }^{2}$ Department of Neurology, University Hospital Giessen and Marburg location Giessen, Klinikstrasse 33, 35385 Giessen, Germany. ${ }^{3}$ Department of Neurology, University Hospital Giessen and Marburg Location Marburg, Baldingerstrasse, 35033 Marburg, Germany. ${ }^{4}$ Department of Anesthesiology, Johannes Gutenberg-University, University Medical Hospital Mainz, Langenbeckstr. 1, 55131 Mainz, Germany.

Received: 19 November 2018 Accepted: 18 December 2018 Published online: 09 January 2019

\section{References}

1. Lv S, Guo ZN, Jin H, Sun X, Jia M, Ma H, et al. Compromised dynamic cerebral autoregulation in patients with epilepsy. Biomed Res Int. 2018;2018: 6958476.

2. Czosnyka M, Smielewski P, Kirkpatrick P, Menon DK, Pickard JD. Monitoring of cerebral autoregulation in head-injured patients. Stroke. 1996;27:1829-34.

3. Sorrentino E, Budohoski KP, Kasprowicz M, Smielewski P, Matta B, Pickard JD, et al. Critical thresholds for transcranial Doppler indices of cerebral autoregulation in traumatic brain injury. Neurocrit Care. 2011;14:188-93.

4. Bode $\mathrm{H}$. Intracranial blood flow velocities during seizures and generalized epileptic discharges. Eur J Pediatr. 1992:151:706-9.

5. Merceron S, Geeraerts T, Montlahuc C, Bedos JP, Resche-Rigon M, Legriel S. Assessment of cerebral blood flow changes in nonconvulsive status epilepticus in comatose patients: a pathophysiological transcranial Doppler study. Seizure. 2014;23:284-9. 